\title{
Comportamento Reprodutivo de Ceratitis capitata Wiedemann (Diptera: Tephritidae): Efeito do Tamanho dos Machos Sobre o seu Sucesso de Cópula
}

\author{
Alberto Moreira Silva Neto ${ }^{\bowtie}$, Vanessa Simões Dias $^{2} \&$ Iara Sordi Joachim-Bravo²
}

1. Universidade Estadual de Feira de Santana, e-mail: bio.alberto@gmail.com (Autor para correspondência ${ }^{\bowtie}$ ). 2. Universidade Federal da Bahia, e-mail:vanessasidias@gmail.com, iara_bravo@yahoo.com.br.

\section{EntomoBrasilis 5 (3): 190-197(2012)}

Resumo. Neste trabalho avaliou-se a influência do tamanho de machos de Ceratitis capitata (Wiedemann) no seu sucesso de cópula. Para assegurar a produção de machos adultos de diferentes tamanhos (grandes e pequenos), dois grupos de larvas foram alimentados com diferentes concentrações de proteína. Subsequentemente, machos adultos de ambos os grupos foram comparados em termos de seu sucesso de cópula (estimado pela capacidade de ser escolhido pela fêmea) e de quantidade de machos que emitiram feromônio. O sucesso de cópula em laboratório foi avaliado com machos em várias proporções, nas quais se manteve constante o número de machos grandes com cinco dias de idade (um único macho) em relação a um aumento progressivo de machos pequenos com mesma idade. As proporções testadas foram 1:1, 1:2, 1:3, 1:4, 1:5, e 1:10. Nos experimentos de sucesso de cópula na proporção 1:1 e nos de emissão de feromônio, foram testados machos pequenos de diferentes idades (cinco, nove ou 13 dias), enquanto que a idade dos machos grandes se manteve constante (cinco dias). Experimentos de sucesso de cópula na proporção 1:1 também foram realizados em gaiola de campo. Evidenciou-se que os machos grandes levaram vantagem em todos os parâmetros analisados em laboratório, emitindo mais feromônio e tendo um maior sucesso de cópula, mesmo quando a idade dos machos pequenos foi variada. O efeito de tamanho foi tão significativo, que na proporção de 1 macho grande para 10 machos pequenos, as fêmeas ainda escolheram os machos grandes. Em gaiola de campo os resultados foram similares aos de laboratório.

Palavras-Chave: Corte; Mosca-das-frutas; Seleção sexual.

\section{Reproductive Behavior of Ceratitis capitata (Wiedemann) (Diptera: Tephritidae): Effect of the Male Size on the Copula Success}

Abstract. This work evaluated the influence of size on the copula success in Ceratitis capitata (Wiedemann). To assure the production of different adult sizes (big and small), two groups of larvae had been fed with different protein concentrations. Subsequently, adult males of both groups had been compared in terms of copula success and amount of males who showed the first step of courtship (emission of sexual pheromone). The copula success in laboratory was evaluated with males in some ratios, which the number of big males with five days of life (an unique male) in relation to a gradual increase of small males with same age kept constant. The tested ratios had been 1:1, 1:2, 1:3, 1:4, 1:5, and 1:10. In the experiments of copula success in the 1:1 ratio and the ones of pheromone emission, they had been tested small male of different ages (five, nine or 13 days), whereas the age of the big males kept constant (five days). Experiments of copula success in the 1:1 ratio had been also carried through in field cage. It was prove that the big males had taken advantage in all the parameters analyzed in laboratory, emitting pheromone and having a bigger copula success, exactly when the age of the small males was varied. The size effect was so significant, that in the ratio of 1 big male for 10 small males, the females had still chosen the big males. In field cage, the results had been similar to the ones of laboratory.

Keywords: Courtship; Fruit Fly; Sexual Selection.

$\mathbf{M}$ uitas espécies de vertebrados e invertebrados possuem um sistema de acasalamento em lek, no qual machos defendem certo território visitado pelas fêmeas, com o único objetivo de copularem. (HöGLund \& ATALO 1995; SHELLY \& WhitTIER 1997). Entre os insetos a maioria dos comportamentos de agregação em leks ocorre na ordem Díptera, mais precisamente na família Tephritidae (Shelly \& Whittier 1997). A mosca das frutas Ceratitis capitata (Wiedemann) (Diptera: Tephritidae), que é uma praga agrícola de importância mundial (MALAVASI et al. 1980), possui seu sistema de acasalamento baseado em leks. (Prokopy \& Hendrichs 1979; Arita \& Kaneshiro 1989; WhitTier et al. 1992). Esses leks contêm entre 2 à 10 machos, os quais soltam uma gota de feromônio sexual a partir de suas respectivas glândulas anais evertidas, para atraírem as fêmeas. (FLATH et al. 1993; Eberhard 1999). O sucesso de cópula de um macho de $C$. capitata depende da sua presença no lek e, posteriormente, dele ser escolhido pela fêmea entre todos os machos ali presentes para copular (KASPI et al. 2000).
Os machos presentes nos leks podem variar muito quanto a vários fatores que podem ser importantes no momento dos mesmos serem selecionados pelas fêmeas. Dentre os padrões biométricos pode-se citar: o tamanho do corpo, comprimento (asas e tórax) e largura (olhos e face) de determinadas partes do corpo, intensidade de emissão de feromônio, posição no lek e também o forrageamento por alimento antes da sua entrada no lek. (Thorhill \& AlCock 1983; HöGlund \& Atalo 1995; Blay \& Yuval 1997; Shelly \& WhitTier 1997; KaSPI et al. 2000; KaSPI \& Yuval 2000; Rodriguero et al. 2002).

Nos insetos, de modo geral, o tamanho do corpo é um fator importante na competitividade reprodutiva do adulto, o qual pode influenciar na capacidade de dispersão, na escolha do parceiro e no sucesso de acasalamento. (SLAnsky \& Scriber 1985). Em insetos holometábolos, essa característica, assim como o tempo de desenvolvimento e amadurecimento sexual, são fortemente influenciadas pela ingestão de proteína na fase imatura (Zucoloto 1988; BRUZzone et al. 1990; ECONOMOPOULOs et al. 1990; KaSPI et 
al. 2002), o que indica que a qualidade nutricional na fase larval também deve ser um fator relevante no sucesso de cópula dos machos.

Em C. capitata a questão dos machos maiores serem sexualmente mais competitivos e atraírem mais fêmeas em relação aos machos menores têm sido bastante discutidas (PARTRIDGE \& FARQUHAR 1983), especialmente em criação massal (BURK \& WEBB 1983; Churchill-Stanland et al. 1986). No entanto, os efeitos do tamanho do macho de $C$. capitata sobre a receptividade da fêmea para cópula, ainda são controversos. Uma série de estudos demonstram uma ampla vantagem de sucesso de cópula para os machos maiores (CHURCHILL-STANLAND et al. 1986; BLAY \& YUVAL 1997; TAYLOR \& YUVAL 1999), entretanto, em outros trabalhos esta vantagem não foi estabelecida (ARITA \& KANESHIRO 1988; WhitTIER et al. 1994; WhITTIER \& KANESHIRO 1995).

Um método de controle da espécie $C$. capitata utilizado em larga escala em algumas partes do mundo é a técnica do inseto estéril (Hendrichs et al. 1995). Para que essa técnica tenha sucesso é fundamental que os machos estéreis de laboratório, soltos na natureza, consigam competir com sucesso com os machos selvagens e copulem com as fêmeas selvagens (KNipLing et al 1989). Dessa forma, estudos que analisem os fatores que influenciam o sucesso de cópula dos machos de $C$. capitata são de fundamental importância.

Neste trabalho analisou-se o efeito do tamanho dos machos de C. capitata sobre o seu sucesso de cópula com as fêmeas sob condições de laboratório e em gaiola de campo.

\section{MATERIAL E MÉTODOS}

A população de $C$. capitata utilizada nos experimentos foi mantida sob condições de laboratório, sem a introdução de moscas selvagens desde 1980, de acordo com Zucoloto (1987). Os adultos receberam diariamente água e uma dieta artificial contendo 6,5 g de lêvedo (Mãe-Terra, Mãe-Terra Produtos Naturais Ltda.), 11,o g de açúcar (União, União de Refinadores do Brasil), 2,o g de agar-agar (Isofar, Isofar Indústria e Comércio de Produtos Químicos Ltda.), 1,o g ácido cítrico (Vetec, Vetec Química Fina Ltda.), 1,o g de nipagin (Isofar), e $100 \mathrm{~mL}$ de água destilada (Zucoloto et al. 1979).

Para obtenção de machos adultos de diferentes tamanhos, dois grupos de larvas foram criados em uma de duas dietas contendo concentrações distintas de lêvedo (fonte com 40\% de sua constituição formada por proteína): uma com 7,o g de lêvedo por $100 \mathrm{~mL}$ de dieta (dieta de alta concentração protéica com 2,3\% de proteína total) e outra com 3,0 g de lêvedo por $100 \mathrm{~mL}$ de dieta (dieta de baixa concentração protéica com 1,1\% de proteína total). Todos os outros componentes foram mantidos nas mesmas proporções da dieta dos adultos.

Todas as larvas eram retiradas da dieta e colocadas na areia para empuparem após sete dias a sua eclosão, que é o tempo médio observado para a duração do estágio larval dos imaturos criados na dieta de alta concentração de proteína. Esse procedimento foi realizado para evitar que as larvas mantidas na dieta de baixa concentração de lêvedo conseguissem compensar este déficit de proteína, passando mais tempo ingerindo dieta e emergissem com tamanho similar aos oriundos da dieta de 7,o g de lêvedo.

Machos recém-emergidos, oriundos da dieta de alta concentração protéica, foram denominados de "machos grandes" e os da dieta de baixa concentração foram designados "machos pequenos".

Em todos os experimentos, machos recém-emergidos, de ambos os grupos foram separados em gaiolas plásticas $(16 \times 11 \times 10 \mathrm{~cm})$ nas quais receberam um tratamento idêntico ao dos adultos da criação. Os machos grandes foram mantidos nessas gaiolas plásticas por quatro dias, enquanto que os machos pequenos foram mantidos por quatro, oito ou doze dias dependendo do experimento que foi realizado. As fêmeas foram provenientes de uma dieta com a mesma concentração protéica dos machos grandes e receberam o mesmo tratamento desses.

Machos de cada grupo foram previamente marcados, na porção dorsal do tórax com tinta atóxica de diferentes cores, 24 horas antes de cada experimento, para futura identificação. As cores foram alternadas em cada repetição.

Os experimentos foram divididos em duas partes: experimentos de laboratório e de gaiola de campo.

Experimentos de laboratório. Em laboratório foram feitos testes de seleção sexual da fêmea entre machos de diferentes tamanhos e testes de comparação do número de machos de diferentes tamanhos que emitiam feromônio sexual. Todos os experimentos foram conduzidos em condições controladas (25$26^{\circ} \mathrm{C}$ e $70 \%$ UR).

\section{Seleção sexual.}

1a) Escolha da fêmea na proporção de machos 1:1. Nesse experimento dois machos adultos (um grande com cinco dias de idade e outro pequeno com cinco, nove ou treze dias de idade) foram colocados em uma caixa plástica redonda $(10 \times 5 \mathrm{~cm})$ juntamente com uma fêmea bem nutrida na fase larval com cinco dias de idade. Foram testados machos pequenos de diferentes idades, porque se sabe que a deficiência proteica na fase imatura, além de causar uma diminuição de tamanho, também pode causar o retardamento da maturação sexual (KASPI et al. 2002). A ocorrência de cópulas foi monitorada a cada cinco minutos das 08:00 às 15:0oh durante dois dias consecutivos. A finalização dos experimentos ocorreu as 15:0oh, porque em observações prévias notaram-se poucas ou nenhuma cópula em laboratório a partir desse horário. Cada casal em cópula foi retirado para identificação do macho. Foram feitas 100 repetições com gerações alternadas.

1b) Os testes com diferentes proporções de machos foram realizados quando as fêmeas, no experimento anterior, exibiram preferência sexual por machos grandes de 5 dias de idade em relação a machos pequenos de cinco dias. A montagem desse experimento seguiu a metodologia empregada no experimento 1a, com a única diferença de que, em cada caixa experimental, era colocado um único macho do grupo preferido no experimento 1a e adicionava-se uma proporção crescente de machos do grupo não preferido. Esses experimentos foram realizados para determinar a capacidade da fêmea escolher o macho do grupo preferido dentro de um conjunto crescente de machos do grupo não preferido. As proporções (macho grande: macho pequeno) testadas foram: 1:2, 1:3, 1:4, 1:5, 1:10. Para cada proporção foram realizadas 30 repetições com gerações alternadas.

2. Emissão de feromônio. As frequências de emissão de feromônio sexual entre machos dos dois grupos de tamanhos foram comparadas em experimentos realizados em uma gaiola de laboratório disposta sobre uma bancada. A gaiola $(68 \times 68 \times 90 \mathrm{~cm})$ era feita de PVC, revestida com tela, e apresentava uma abertura lateral para a entrada dos braços e cabeça do observador. Dentro dela era acondicionada uma muda de pitanga [Eugenia uniflora (Linnaeus)] envasada, com $60 \mathrm{~cm}$ de altura e $40 \mathrm{~cm}$ de raio de copa e pedaços de maçã [Malus domestica (Borkh)] dispostos nas extremidades dos galhos. Quarenta machos, previamente marcados, sendo vinte grandes com cinco dias de idade e vinte pequenos com cinco dias de idade, foram acondicionados nesta gaiola e monitorados quanto à emissão de feromônio por um período de uma hora (08:0oh as 09:0oh). Esse horário foi escolhido por ter sido considerado o período de pico de ocorrência de cópulas, em experimentos preliminares. Cada macho que liberava a gota de feromônio era retirado para identificação e posterior medição do tamanho da asa. Após a finalização do experimento, os machos que não emitiram feromônio, também foram fixados e medidos. Foram feitas cinco repetições com gerações alternadas. A metodologia para testar cada uma das 
comparações entre o número de machos grandes de cinco dias e os machos pequenos com nove ou 13 dias que emitiam feromônio foi a mesma descrita acima. A única diferença é que, nesse caso, foram comparados machos grandes de cinco dias com relação a machos pequenos com nove dias de idade e machos grandes de cinco dias com relação a machos pequenos com 13 dias de idade.

Experimentos de gaiola de campo. Esses experimentos foram realizados em gaiola de campo (230 x $150 \times 230 \mathrm{~cm})$, no Campus de Ondina da Universidade Federal da Bahia, Salvador/ Bahia, no período entre janeiro de 2006 a janeiro de 2007. Os experimentos foram montados somente em dias ensolarados e a temperaturas variaram entre 26 a $37^{\circ} \mathrm{C}$ e a umidade relativa entre $60 \%$ e $77 \%$.

Seleção sexual em gaiola de campo. Foram utilizadas gaiolas (230 x $150 \times 230 \mathrm{~cm}$ ), nas quais era acondicionada uma muda de pitanga (E. uniflora), com, aproximadamente, $107 \mathrm{~cm}$ de altura e raio de copa em torno de $40 \mathrm{~cm}$, colocada sobre um banco de madeira com $60 \mathrm{~cm}$ de altura, com pedaços de maçã (M. domestica) dispostos nas extremidades dos galhos. Nessas gaiolas foram introduzidos 100 machos (50 grandes com cinco dias de idade e 50 pequenos com cinco dias de idade de idade, previamente marcados por tinta atóxica com cores distintas), juntamente, com 50 fêmeas de cinco dias de idade, oriundas da dieta de $7 \mathrm{~g}$ de lêvedo. A ocorrência de cópulas foi monitorada a cada cinco minutos, das 08:0oh às 13:0oh. Cada casal em cópula era gentilmente retirado para identificação do macho e posterior medição do tamanho da asa. Sempre que um casal era retirado observava-se a origem do macho em cópula e retiravase um macho do grupo oposto. Este procedimento visou manter dentro da gaiola sempre a proporção de machos com diferentes tamanhos em 1:1. Foram feitas cinco repetições com gerações alternadas. Ao final do experimento todos os machos que não copularam também foram coletados e fixados para medidas biométricas. A metodologia utilizada para testar cada uma das comparações entre o número de machos grandes de cinco dias e os machos pequenos com 9 ou 13 dias que emitiam feromônio foi a mesma descrita acima, com a única diferença que nesses testes foram comparados machos grandes de cinco dias com relação a machos pequenos com nove dias de idade e machos grandes de cinco dias com relação a machos pequenos com 13 dias de idade.
Medidas biométricas. Ao final dos experimentos todos os machos foram fixados em álcool a 70\%, para medição do tamanho da asa esquerda (distância entre a nervura R4+5 a m-cu), como estimativa do tamanho do corpo (Zucoloto 1987). Estas medidas foram feitas com auxílio de um estereomicroscópio munido de ocular micrométrica com conversão de 0,5 $\mathrm{mm}$ em um aumento de 20 vezes.

Análise estatística. Todos os dados dos experimentos de seleção sexual em laboratório foram analisados usando o teste do Qui-quadrado $\left(\chi^{2}\right)$ com auxílio do programa StatSoft, Inc. (2001). STATISTICA (data analysis software system), version 6. A frequência de cópulas esperada para cada tipo de macho (Esperado) utilizada em todos os teste de Qui-quadrado foi calculada da seguinte forma: Esperado $=\mathrm{Nr} / \mathrm{TM} \times \mathrm{Mi}$, na qual "Nr" é número total de repetições, "TM" o número total de machos dentro de cada caixa experimental e "MI" número de machos do tipo em interesse dentro de cada caixa do experimento. Para análise dos dados de emissão de feromônio e de seleção sexual em gaiola de campo, foi utilizado o teste t não pareado, com o auxílio do programa GraphPad InStat version 3.00 for Windows 95, GraphPad Software, San Diego California USA. Para comparação entre o tamanho das asas dos diferentes grupos de idades em cada experimento, utilizou-se também o teste $t$ não pareado. Todos os dados, nos quais o teste $t$ foi aplicado, passaram no teste de normalidade de Kolmogorov e Smirnov. Em alguns casos nos quais os dois grupos comparados não possuíam homogeneidade de variâncias, a correção de Welch foi aplicada para possibilitar a utilização do teste t. Em todos os testes estatísticos utilizados assumiu-se o alfa de $5 \%$.

\section{RESULTADOS}

Seleção sexual em laboratório. Nesses experimentos (Figura 1), as fêmeas preferiram copular com os machos grandes de 5 dias em relação aos machos pequenos de 5, 9 ou 13 dias de idade (teste do Qui-quadrado: grande 5 dias versus pequeno 4 dias $\chi^{2}=81,00 \mathrm{df}=1 \mathrm{p}<0,00001-($ Figura 1a); grande 4 dias versus pequeno 8 dias $\chi^{2}=51,84 \mathrm{df}=1 \mathrm{p}<0,00001-($ Figura $1 \mathrm{~b})$ e grande 4 dias versus pequeno de 12 dias $\chi^{2}=77,44 \mathrm{df}=1 \mathrm{p}<$ 0,00001 - (Figura 1c).
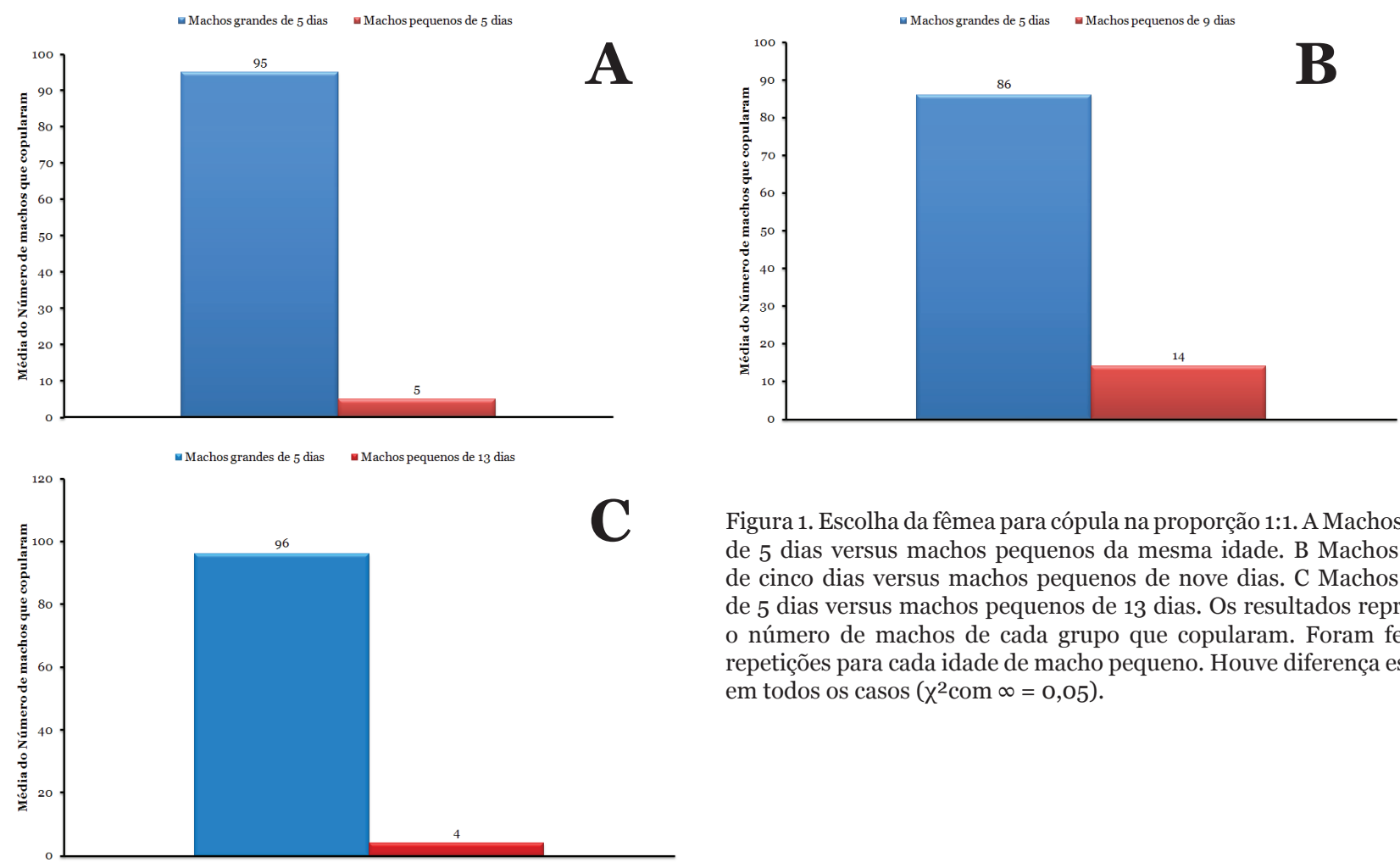

Figura 1. Escolha da fêmea para cópula na proporção 1:1. A Machos grandes de 5 dias versus machos pequenos da mesma idade. B Machos grandes de cinco dias versus machos pequenos de nove dias. C Machos grandes de 5 dias versus machos pequenos de 13 dias. Os resultados representam o número de machos de cada grupo que copularam. Foram feitas 100 repetições para cada idade de macho pequeno. Houve diferença estatística em todos os casos $\left(\chi^{2} \operatorname{com} \infty=0,05\right)$. 
Como ocorreu uma preferência da fêmea para copular com os machos grandes de 5 dias em relação aos machos pequenos de mesma idade, na proporção 1:1 (grande:pequeno), foram realizados os experimentos de escolha da fêmea entre um macho grande de 5 dias frente a uma proporção crescente de machos pequenos, também de 5 dias. Em todas as proporções (macho grande: macho pequeno) testadas, as fêmeas preferiram copular com os machos grandes em relação aos pequenos (Figura 2) (teste do Qui-quadrado - proporção 1:2, $\chi^{2}=38$, $40 \mathrm{df}=1 \mathrm{p}<$ 0,00; proporção 1:3, $\chi^{2}=67,60 \mathrm{df}=1 \mathrm{p}<0,00$; proporção 1:4, $\chi^{2}$ $=67,50 \mathrm{df}=1 \mathrm{p}<0,00 ;$ proporção $1: 5, \chi^{2}=77,760 \mathrm{df}=1 \mathrm{p}<0,00 \mathrm{e}$ proporção 1:10, $\left.\chi^{2}=134,51 \mathrm{df}=1 \mathrm{p}<0,00\right)$.

As medidas biométricas evidenciaram que as medidas das asas dos machos grandes sempre foram maiores do que as dos machos pequenos (Tabela 1), confirmando a diferença de tamanho dos machos confrontados.

Emissão de feromônio. Os resultados desses experimentos mostraram que o número de machos grandes de cinco dias que emitiram feromônio foi superior ao de machos pequenos de cinco, nove ou 13 dias de idade, que efetuaram esse comportamento (Figura 3) (teste T, para dados não pareado: machos grandes de cinco dias versus machos pequenos de cinco dias, $\mathrm{p}=0,0014$; machos grandes de cinco dias versus machos pequenos de nove dias, $\mathrm{p}=0,0008$; machos grandes de cinco dias versus machos pequenos de 13 dias, $\mathrm{p}=0,0067)$.

Em todas as gaiolas de emissão de feromônio, os machos grandes sempre apresentaram asas maiores do que os machos pequenos (teste T, para dados não pareado) (Tabela 2).

Seleção sexual em gaiola de campo. Em gaiolas de campo (Figura 4) as fêmeas também preferiram copular com os machos grandes de cinco dias em relação aos machos pequenos de mesma idade (teste $\mathrm{T}$, para dados não pareado a $5 \%$ de significância; $\mathrm{p}$ $=0,0003$ ).

Assim como nos outros experimentos, os machos grandes testados sempre apresentaram asas maiores do que os machos pequenos (teste t não pareado) (Tabela 3).

Tabela 1. Medida da asa (mm) como estimativa do tamanho do corpo dos machos de diferentes grupos de tamanho, nos testes de seleção sexual em laboratório. Os resultados representam as médias e os desvios padrão do tamanho da asa (medida da distância da nervura R4+5 a m-cu) em milímetros. (Teste $t$ não pareado com correção de Welch). $\mathrm{G}=$ machos grandes e $\mathrm{P}=$ machos pequenos.

\begin{tabular}{cccc}
\hline & Tamanho da Asa (mm) & \\
\hline Proporções & Machos grandes & Machos pequenos & Valor do p \\
\hline 1:1 G5dias x P5dias & $2,7 \pm 0,07$ & $2,2 \pm 0,15$ & $<0,0001$ \\
$1: 1$ G5dias x P9dias & $2,7 \pm 0,06$ & $2,2 \pm 0,12$ & $<0,0001$ \\
$1: 1$ G5dias x P13dias & $2,7 \pm 0,06$ & $2,2 \pm 0,12$ & $<0,0001$ \\
$1: 2$ G5dias x P5dias & $2,7 \pm 0,08$ & $2,2 \pm 0,15$ & $<0,0001$ \\
$1: 3$ G5dias x P5dias & $2,7 \pm 0,12$ & $2,2 \pm 0,14$ & $<0,0001$ \\
$1: 4$ G5dias x P5dias & $2,7 \pm 0,09$ & $2,2 \pm 0,12$ & $<0,0001$ \\
$1: 5$ G5dias x P5dias & $2,7 \pm 0,19$ & $2,3 \pm 0,14$ & $<0,0001$ \\
$1: 10$ G5dias x P5dias & $2,7 \pm 0,19$ & $2,3 \pm 0,15$ & $<0,0001$ \\
\hline
\end{tabular}

Tabela 2. Medida da asa ( $\mathrm{mm}$ ) como estimativa do tamanho do corpo dos machos de diferentes grupos de tamanho, nos testes de emissão de feromônio sexual em laboratório. Os resultados representam as médias e os desvios padrão do tamanho da asa (medida da distância da nervura R4+5 a m-cu) em milímetros. (Teste t não pareado com correção de Welch). $\mathrm{G}=$ machos grandes e $\mathrm{P}=$ machos pequenos.

\begin{tabular}{|c|c|c|c|}
\hline \multicolumn{4}{|c|}{ Tamanho da Asa (mm) } \\
\hline Gaiolas & Machos grandes & Machos pequenos & Valor do p \\
\hline \multicolumn{4}{|c|}{ Machos grandes de 5 dias versus Machos pequenos de 5 dias } \\
\hline 1 & $2,7 \pm 0,07$ & $2,3 \pm 0,09$ & $<0,0001$ \\
\hline 2 & $2,7 \pm 0,06$ & $2,1 \pm 0,10$ & $<0,0001$ \\
\hline 3 & $2,7 \pm 0,06$ & $2,2 \pm 0,10$ & $<0,0001$ \\
\hline 4 & $2,7 \pm 0,08$ & $2,2 \pm 0,12$ & $<0,0001$ \\
\hline 5 & $2,7 \pm 0,07$ & $2,2 \pm 0,15$ & $<0,0001$ \\
\hline \multicolumn{4}{|c|}{ Machos grandes de 5 dias versus Machos pequenos de 9 dias. } \\
\hline 1 & $2,7 \pm 0,07$ & $2,3 \pm 0,13$ & $<0,0001$ \\
\hline 2 & $2,7 \pm 0,06$ & $2,2 \pm 0,11$ & $<0,0001$ \\
\hline 3 & $2,7 \pm 0,09$ & $2,2 \pm 0,13$ & $<0,0001$ \\
\hline 4 & $2,7 \pm 0,13$ & $2,2 \pm 0,13$ & $<0,0001$ \\
\hline 5 & $2,7 \pm 0,06$ & $2,2 \pm 0,14$ & $<0,0001$ \\
\hline \multicolumn{4}{|c|}{ Machos grandes de 5 dias versus Machos pequenos de 13 dias. } \\
\hline 1 & $2,7 \pm 0,06$ & $2,2 \pm 0,08$ & $<0,0001$ \\
\hline 2 & $2,7 \pm 0,06$ & $2,2 \pm 0,12$ & $<0,0001$ \\
\hline 3 & $2,7 \pm 0,05$ & $2,2 \pm 0,09$ & $<0,0001$ \\
\hline 4 & $2,7 \pm 0,04$ & $2,3 \pm 0,09$ & $<0,0001$ \\
\hline 5 & $2,8 \pm 0,05$ & $2,2 \pm 0,08$ & $<0,0001$ \\
\hline
\end{tabular}

Tabela 3. Medida da asa (mm) como estimativa do tamanho do corpo dos machos de diferentes grupos de tamanho, nos testes de seleção sexual em gaiola de campo. Os resultados representam as médias e os desvios padrão do tamanho da asa (medida da distância da nervura R4+5 a m-cu) em milímetros. (Teste t não pareado com correção de Welch). $\mathrm{G}=$ machos grandes e $\mathrm{P}=$ machos pequenos.

\begin{tabular}{|c|c|c|c|}
\hline \multicolumn{4}{|c|}{ Tamanho da Asa (mm) } \\
\hline Gaiolas & Machos grandes & Machos pequenos & Valor do p \\
\hline 1 & $2,8 \pm 0,06$ & $2,2 \pm 0,11$ & $<0,0001$ \\
\hline 2 & $2,7 \pm 0,06$ & $2,3 \pm 0,13$ & $<0,0001$ \\
\hline 3 & $2,7 \pm 0,09$ & $2,3 \pm 0,11$ & $<0,0001$ \\
\hline 4 & $2,8 \pm 0,06$ & $2,3 \pm 0,11$ & $<0,0001$ \\
\hline 5 & $2,7 \pm 0,09$ & $2,3 \pm 0,10$ & $<0,0001$ \\
\hline
\end{tabular}




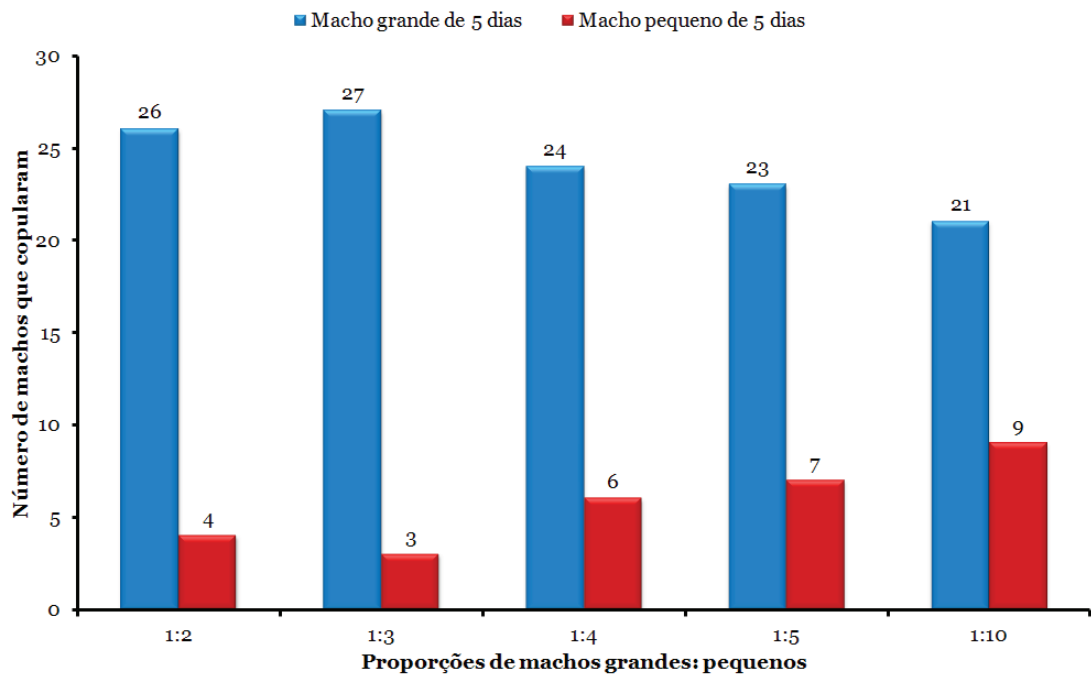

Figura 2. Capacidade da fêmea para escolher (avaliado pela aceitação de cópula) o macho grande frente a um crescente número de machos pequenos. Os resultados representam o número de cópula dos machos de cada grupo de tamanho em 30 repetições para cada proporção. Todos os machos testados tinham cinco dias de idade. Houve diferença estatística em todas as proporções $\left(\chi^{2}\right.$ com $\left.\infty=0,05\right)$.
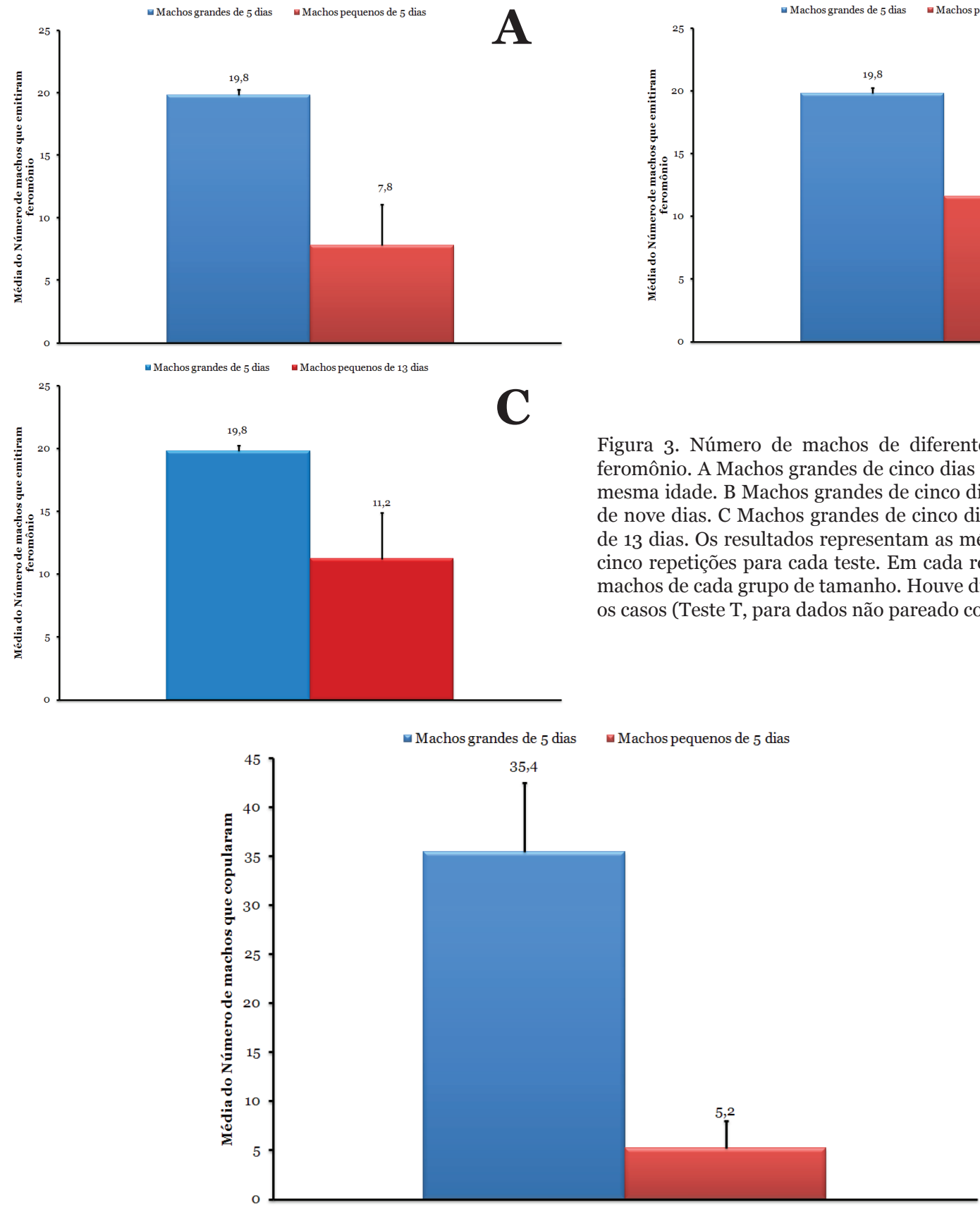

Figura 4. Seleção sexual em gaiola de campo. Escolha das fêmeas entre machos grandes e pequenos de 5 dias de idade. Os resultados representam as médias e os desvios padrão de 5 repetições. Cada repetição continha 50 machos de cada grupo de tamanho. Houve diferença estatística $\mathrm{p}=0,0003$ (Teste T, para dados não pareado $\operatorname{com} \infty=0,05$ ).

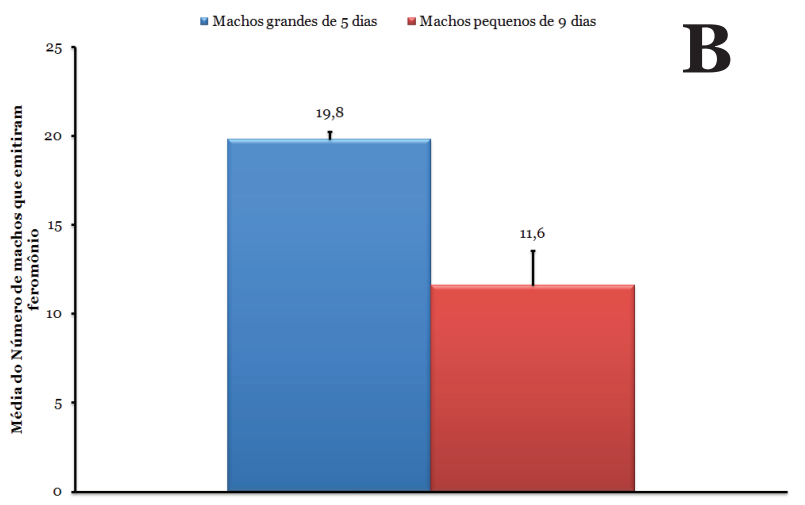

Figura 3. Número de machos de diferentes tamanhos que emitiram feromônio. A Machos grandes de cinco dias versus machos pequenos da mesma idade. B Machos grandes de cinco dias versus machos pequenos de nove dias. C Machos grandes de cinco dias versus machos pequenos de 13 dias. Os resultados representam as médias e os desvios padrão de cinco repetições para cada teste. Em cada repetição foram avaliados 20 machos de cada grupo de tamanho. Houve diferença estatística em todos os casos (Teste T, para dados não pareado com $\alpha=0,05$ ). 


\section{DISCUSSÃO}

Nos experimentos de seleção sexual em laboratório, as fêmeas de C. capitata apresentaram uma preferência para cópula com os machos maiores de cinco dias de idade frente aos machos menores de cinco dias. Este dado corrobora outros estudos realizados em laboratório, nos quais as moscas utilizadas também pertenciam a linhagens mantidas em cativeiro por várias gerações. (CHURCHILLStANLAND et al. 1986; BLAY \& Yuval 1997; TAYlor \& Yuval 1999) e indica que o tamanho é um fator importante para a escolha da fêmea.

Como tempo de maturação sexual de machos alimentados na fase larval com dietas de baixa concentração protéica tende a ser maior do que o de machos alimentados adequadamente nessa fase (KASPI et al. 2002), seria possível que a preferência das fêmeas por machos maiores de cinco dias de idade em relação aos menores de mesma idade, fosse em função da maturação tardia dos últimos e não por causa do tamanho em si. Entretanto, os resultados evidenciaram que as fêmeas continuaram a escolher os machos maiores de cinco dias de idade, mesmo quando estes foram confrontados com machos menores de nove ou 13 dias, que já estariam maduros sexualmente. Tais dados reforçam a indicação de que a escolha das fêmeas tenha sido pelo fator tamanho em si e não por variações no tempo de maturação sexual entre os grupos de machos testados.

Nos experimentos de seleção sexual, sob condições de gaiola de campo, as fêmeas também mantiveram sua preferência para copular com os machos maiores de cinco dias em relação aos menores de mesma idade. Resultado semelhante foi obtido em outro estudo, com moscas também criadas em cativeiro a várias gerações e realizado em gaiola de campo, no qual as fêmeas também preferiram copular com os machos maiores (OROzco \& LóPEz 1993). Porém, no mesmo trabalho quando foi utilizada uma população de moscas selvagens, não se obteve uma correlação positiva entre o tamanho do macho e o seu sucesso de cópula. RoDriguero et al. (2002) realizando estudos de seleção sexual em gaiola de campo, encontrou um correlação positiva entre o tamanho da asa dos machos e o sucesso de cópulas dos mesmos, tanto na linhagem de machos de laboratório como nos machos selvagens. Outros estudos realizados em campo com linhagens de laboratório contradizem os resultados do presente trabalho, pois neles, não existiu um efeito do tamanho do macho sobre o seu sucesso de cópula. (ARITA \& KANESHIRo 1988; WhitTIER et al. 1994; WhitTIER \& KANESHIRO 1995). Cabe lembrar que na maioria dos trabalhos citados acima, o fator tamanho foi analisado, muitas vezes, em conjunto com o fator alimentação na fase adulta com privação ou não de proteína e, talvez, o efeito da alimentação nesta fase tenha influenciado na falta de correlação entre o tamanho e o sucesso reprodutivo.

No presente trabalho todos os machos na fase adulta receberam uma dieta contendo uma concentração proteica adequada para um bom desenvolvimento fisiológico segundo Zucoloto et al. (1979), com a finalidade de evitar efeitos no sucesso de cópula por variação da dieta do adulto analisando-se apenas o efeito do tamanho.

Asimilaridade obtida entre os resultados de campo edelaboratório demonstrou que experimentos realizados em laboratório podem refletir bem futuros experimentos de campo. A realização de experimentos em laboratório, além disso, permite uma maior manipulação do fator em análise, através do controle de variáveis de confusão, tais como temperatura e luminosidade (HENDRICHS \& HENDRICHS 1990).

O sucesso de cópula de um macho de $C$. capitata depende da sua presença no lek e da sua capacidade de ser escolhido pela fêmea, sendo que, o sucesso desse último passo depende, além de fatores morfológicos e fisiológicos, da realização de uma corte adequada (KASPI et al. 2000). O comportamento de corte dos machos de $C$. capitata, é amplamente conhecido, passando por estágios bem definidos. O primeiro deles é a emissão de feromônio de atração das fêmeas a longa distância, com a elevação da parte posterior de seu abdome e liberação de feromônio, através das glândulas anais eversíveis (Limmatainen et al. 1997; Calcagno et al. 1999; BRICEÑO \& EBERHARD 2000; BRICEÑo et al. 2002).

Os testes de emissão de feromônio aqui realizados mostraram que o número de machos grandes com cinco dias de idade, que emitiram feromônio na primeira hora de luz diária foi maior que o de machos pequenos de mesma idade. O mesmo ocorreu quando machos grandes de cinco dias foram confrontados com machos pequenos de nove e 13 dias. Pode-se inferir que uma das possíveis causas da vantagem no sucesso de cópula dos machos grandes com relação aos pequenos, encontrada neste trabalho, pode ter ocorrido devido a um maior investimento por parte dos machos grandes em atrair a fêmea, através da emissão da gota de feromônio nesse período do dia.

As fêmeas demonstraram uma alta capacidade de percepção para cópula com o macho maior, mesmo quando ele estava no meio de vários machos menores. Nos testes de proporção de um macho maior frente a cinco machos menores, por exemplo, o esperado para 30 repetições de escolha da fêmea para cópula era que 25 fêmeas escolhessem os machos menores e apenas cinco fêmeas escolhessem os machos maiores. No entanto, aconteceu quase o inverso, de modo que, 23 fêmeas escolheram os machos maiores e apenas sete fêmeas escolheram os machos menores.

A partir dos resultados dos experimentos de proporção, podese fazer uma inferência que, por exemplo, se o experimento de proporção 1:3 simulasse um lek com quatro indivíduos (três machos pequenos e um macho grande), a fêmea conseguiria perceber o macho maior, talvez até por uma percepção visual, uma vez que estudos demonstraram capacidade visual das fêmeas para reconhecer traços morfométricos dos machos (comprimento da asa, da arista, do tórax , largura da face e da cabeça) durante a corte (MENDEZ et al. 1998; BRICEÑo \& EBERHARD 2002; RodRIGUERO et al. 2002).

A quantidade de machos presentes nos leks já foi alvo de inúmeros estudos demonstrando variações na quantidade de machos presentes nos mesmos. Esses números podem variar de três a quatro machos sob condições naturais até oito a 16 machos após a liberação de machos estéreis no Hawai (SHelly et al. 1994; SHELLY \& WhitTier 1996), de três a seis machos em gaiola de campo na Guatemala (PROKOPY \& HENDRICHS 1979) ou quatro machos após a liberação de machos estéreis no México (BAKER \& VAN-DER-VALK 1992). Sendo assim, seria interessante em cada região e com cada linhagem especifica de macho estéril, após a realização de testes piloto para se determinar a média do número de machos presentes em cada lek, utilizar experimentos de proporções semelhantes aos do presente trabalho, envolvendo machos estéreis e machos selvagens, com a finalidade de se analisar o sucesso de cópula desses machos estéreis em proporções estéreis: selvagens similares àquelas existentes após a liberação em campo.

Um trabalho teórico, sobre a quantidade ideal de machos estéreis que deveriam ser soltos em campo, foi realizado por KNIPLING (1955), no qual ele através de inferências matemáticas calculou que numa área isolada contendo uma população de 2 milhões de $C$. capitata, sendo metade composta por machos, deveriam ser soltos 2 milhões de machos estéreis a cada geração. Isso iria suprimir, a partir da quarta geração, quase toda a população local de $C$. capitata. A proporção utilizada por ele foi de um macho selvagem dois machos estéreis. No mesmo trabalho KNIPLING (1955) recomenda que se utilize a proporção de um macho selvagem para cada nove machos estéreis (1:9). Conhecendo-se bem todos os fatores, que estão ligados ao sucesso de cópula dos machos de $C$. capitata, os experimentos de proporções como aqui realizados, poderiam favorecer a técnica do inseto estéril, servindo de base para se calcular a quantidade de machos estéreis 
que deveria ser liberada em determinada região, economizando recursos financeiros.

Um trabalho realizado por KRAINACKER et al. (1987), no qual, larvas de $C$. capitata foram criadas em 24 tipos diferentes de frutos hospedeiros, evidenciou que os adultos provenientes desses hospedeiros variaram com relação a sua sobrevivência, tempo de desenvolvimento, fecundidade do adulto e tamanho do corpo.

Como as fêmeas de C. capitata são capazes de infestar mais de 250 espécies de plantas (LiQuido et al. 1991) e a qualidade nutricional destes inúmeros frutos hospedeiros varia enormemente, em relação a diversos nutrientes, inclusive a concentração de proteína, pode-se imaginar que na natureza o tamanho dos machos varie bastante. Situações em que ocorre competição de machos com tamanhos diferentes, com a finalidade de copular com a fêmea, tais como no presente trabalho, podem ocorrer na natureza entre os próprios machos selvagens. Um estudo realizado por RODRIGUERo et al. (2002), demonstrou que machos maiores levaram vantagem no seu sucesso de cópula com as fêmeas selvagens, independentemente de serem machos de laboratório ou selvagens.

As dietas oferecidas às larvas em criações de laboratório, geralmente, possuem uma maior quantidade de proteína do que em muitos frutos na natureza (HENDrichs et al. 1993; Murphy et al. 1994). No entanto, é necessário ressaltar que nem sempre isso é garantia de um desempenho melhor dos imaturos que ingerem essas dietas quando comparados, aos que ingerem frutos, uma vez que a digestibilidade e a disponibilidade dos nutrientes nos frutos pode ser mais adequada (JoACHIM-Bravo \& Zucoloto 1998).

No presente trabalho, os machos criados com 2,3\% de proteína foram bem maiores e tiveram um desvio padrão bem menor, quanto ao tamanho, quando comparados aos dos machos criados com $1,1 \%$ de proteína. Tais dados indicam que nas criações em laboratório, que visam a produção de machos estéreis em grande escala, a utilização de dietas que garantam a produção de machos com tamanhos padronizados e bem maiores que os encontrados na natureza são de grande relevância para o sucesso da técnica do inseto estéril, uma vez que, os dados deste trabalho apontam o tamanho do macho como um fator muito importante para o seu sucesso de cópula.

\section{AGRADECIMENTOS}

Agradecemos a Fapesb (Fundação de Amparo a Pesquisa do Estado da Bahia) pelo apoio financeiro para realização deste trabalho dado, sob número do contrato APRoo01/2006; e pela bolsa concedida ao primeiro autor, sob o número de contrato BOL0266/2005.

\section{REFERÊNCIAS}

Arita, L.H. \& K.Y. Kaneshiro, 1988. Body size and differential mating success between males of two populations of the Mediterranean fruit fly. Pacific Science, 42: 173-177.

Arita, L.H. \& Y. Kaneshiro, 1989. Sexual selection and lek behavior in the Mediterranean fruit fly, Ceratitis capitata (Diptera: Tephritidae). Pacific Science, 43: 135-143.

Baker, P.S. \& H. Van der Valk. 1992. Distribution and behaviour of sterile Mediterranean fruit flies in a host tree. Journal Applied Entomology, 114: 67-76.

Blay S. \& B. Yuval, 1997. Nutritional correlates to reproductive success of male Mediterranean fruit flies. Animal Behaviour, 54: 59-66.

Briceño, R.D. \& W.G. Eberhard, 2000. Male wing positions during courtship by Mediterranean fruit flies (Ceratitis capitata) (Diptera: Tephritidae). Journal of the Kansas Entomological Society, 73: 111-115.

Briceño, R.D. \& W.G. Eberhard, 2002. Courtship in the medfl $\mathrm{y}$,Ceratitis capitata, includes tactile stimulation with the male's aristae. Entomologia Experimentalis Applicata .102: 221-228

Briceño, R.D., W.G. Eberhad, J.C. Vilardi \& P. Liedo, 2002. Variation in the intermittent buzzing songs of male medflies (Diptera: Tephritidae: Ceratitis capitata) associated with geography, mass-rearing, and courtship success. Florida Entomologist, 85: 32-40.

Bruzzone, N.D., A.P. Economopoulos \& H.S. Wang, 1990. Mass rearing Ceratitis capitata: reuse of the finisher larval diet. Entomologia Experimentalis Applicata, 27: 103-106.

Burkt, T. \& J.C. Webb, 1983. Effect of male size on calling propensity, song param-eters, and mating success in Caribbean fruit flies (Anastrepha suspensa (Loew). Annals of the Entomological Society of America, 76: 678-682.

Calcagno, G., M.T. Vera, F. Manso, S. Lux, F. Norry, N. Munyiri \& J.C. Vilardi, 1999. Courtship behavior of wild and massrearing Mediterranean fruit fly (Diptera: Tephritidae) males from Argentina. Journal of Economic Entomology, 2: 373379.

Churchill-Stanland, C., R. Stanland; T.Y. Wong, N. Tanaka, D.O. Mcinnis \& R.V. Dowell, 1986. Size as a factor in the mating propensity of Mediterranean fruit flies, Ceratitis capitata (Diptera: Tephritidae), in the laboratory. Journal of Economic Entomology, 79:614-619.

Eberhard, W.G., 1999. Sexual behavior and sexual selection in the medfly, Ceratitis capitata. p. 459-489. In: Aluja, M. \& A. Norrbom (eds). Fruit Flies (Tephritidae): Phylogeny and Evolution of Behavior. CRC Press, Boca Raton, 489p.

Economopoulos A.P., A.A. Al-Taweel \& N.D. Bruzzone, 1990. Larval diet with a starter phase for mass-rearing Ceratitis capitata: substitution and refinement in the use of yeast and sugars. Entomologia Experimentalis Applicata, 55: 239-246.

Flath, R.A., E.B. Jang, D. M. Light, R.T. Mon, L. Carvalho, R.G. Binder \& J.O. John, 1993. Volatile pheromonal emissions from the Mediterranean fruit By. Effects of By age and time of day. Journal of Agricultural an Food Chemistry, 41: 830837.

Hendrichs, J. \& M.A. Hendrichs, 1990. Mediterranean fruit fly (Diptera: Tephritidae) in nature: location and diel pattern of feeding and other activities on fruiting and nonfruiting host and nonhost trees. Annals of the Entomological Society of America, 83: 632-641.

Hendrichs, J., G. Franz \& P. Rendon, 1995. Increased effectiveness and applicability of the sterile insect technique through maleonly releases for control of Mediterranean fruit flies during fruiting seasons. Journal Applied Entomology, 119: 371-377.

Hendrichs, J, V. Wornoayporn, B.I. Katsoyannos \& K. Caggl, 1993. First field assessment of the dispersal and survival of mass reared sterile Mediterranean fruit fly males of an embryonal, temperature sensitive genetic sexing strain, p 453-462 In: Management of Insect Pests: Nuclear and Related Molecular and Genetic Techniques. IAEA, Vienna, Austria, 187p.

Höglund, J. \& R. Alatalo, 1995. Leks. Princeton University Press, Princeton, NJ. 248p.

Joachim-Bravo, I.S. \& F.S. Zucoloto, 1998. Performance and feeding behavior of Ceratitis capitata: comparison of a wild population and laboratory population. Entomologia Experimentalis et Applicata, 87: 67-72.

Kaspi, R. \& B.Yuval,200o.Post-teneral protein feeding improves sexual competitiveness but reduces longevity of mass reared sterile male Mediterranean fruit flies. Annals of the Entomological Society of America, 93: 949-955.

Kaspi R., P.W. Taylor \& B. Yuval, 2000. Diet and size influence sexual advertisement and copulatory success of males in Mediterranean fruit fly leks. Ecological Entomology, 25: 1-6.

Kaspi, R, S. Mossinson, T. Drezner, B. Kamensky \& B. Yuval, 2002. Effect of larval diet on development rates and reproductive maturation of male and female Mediterranean fruit flies. Physiological Entomology, 27: 29-38.

Knipling E.F, C.O Calkins \& T.R. Ashley, 1989. The impact of poor quality of mass-reared Mediterranean fruit flies on 
the sterile insect technique used for eradication. Journal of Applied Entomology, 108: 401-408.

Knipling, E.F., 1955. Possibilities of insect control or eradication through the use of sexual sterile males. Journal of Economic Entomology, 48: 459-462.

Krainacker, D.A., J.R. Carey \& R.I. Vargas, 1987. Effect of larval host on life history traits of the Mediterranean fruit fly, Ceratitis capitata. Oecologia, 73: 583-590.

Liimatainen, J., A. Hoikkala \& T. Shelly, 1997. Courtship behavior in Ceratitis capitata (Diptera: Tephritidae): Comparison of wild and mass-reared males. Annals of the Entomological Society of America, 90: 836-843.

Liquido, N.J., R.T. Cunningham \& S. Nakagawa, 1991. Host plants of the Mediterranean fruit fly (Diptera: Tephritidae): an annotated world review. Miscellaneous Publication. Annals of the Entomological Society of America Lanham, MD, USA. 77p.

Malavasi, A., J.S. Morgante \& R.A. Zucchi, 1980. Biologia da moscas-das-frutas(Diptera:Tephritidae): Listadehospedeiros e ocorrência. Revista Brasileira de Biologia, 40: 9-16.

Mendez, V., R.D. Briceño \& W.G. Eberhad, 1998. Functional significance of the capitate supra-fronto-orbital bristles of male medflies (Ceratitis capitata) (Diptera: Tephritidae). Journal of the Kansas Entomological Society, 71:164-174.

Murphy, T. A, S.C. Loerch \& K.E. McClure, 1994. Effects of grain or pasture finishing systems on carcass composition and tissue accretion rates of lambs. Journal of Animal Science, 72: 3138-3144.

Orozco, D. \& R.O. Lopez, 1993. Mating Competitiveness of wild and laboratory mass-reared med-flies: effect of male size, $p$. 185-188. In: M. Aluja \& P. Liedo [eds.], Fruit Flies: biology and management. Springer, NY. 492p.

Partridge, L \& M. Farquhar, 1983. Lifetime mating success of male frutflies (Drosophila melanogaster) is related to their size. Animal Behaviour, 31: 871-877.

Prokopy, R.J. \& J. Hendrichs, 1979. Mating behavior of Ceratitis capitata on a field-cage host tree. Annals of the Entomological Society of America, 72: 642-648.

Rodrigueiro, M.S., J.C. Vilardi, M.T. Vera, J.P. Cayol \& E. Rial, 2002. Morphometric traits and sexual selection in medfly (diptera: tephritidae) under field cage conditions. Florida Entomologist, 85: 143-149.

Shelly, T.E. \& T.S. Wihttier, 1996. Mating competitiveness of sterile male mediterranean fruit flies (Diptera: Tephritidae) in male-only releases. Annals of the Entomological Society of America, 89: 754-758.
Shelly, T.E., T.S. Whittier \& K.Y. Kaneshiro, 1994. Sterile insect release and the natural mating system of the Mediterranean fruit By, Ceratitis capitata (Diptera: Tephritidae). Annals of the Entomological Society of America, 87: 470-481.

Shelly, T. \& T S. Wihttier, 1997. Lek behavior of insect. Mating Systems, p. 273-293. In: J.C. Choe \& B. J. Crespi (Eds). The evolution of mating systems in Insect and Arachnids . Cambridge. Cambridge University Press, 397p.

Slansky, F. \& J.M. Scriber, 1985. Food consumption and utilization, p 89-163. In: G. A. and Gilbert, L. I. (Eds.). Kerkut, Comprehensive InsectPhysiology, Biochemistry and Pharmacology Vol. 4, Pergamon Press, Oxford, 594P.

StatSoft Inc., 2001. STATISTICA (Data Analysis Software System), versão 6. Disponível em: <www.statsoft.com>.

Taylor, P.W. \& B. Yuval, 1999. Postcopulatory sexual selection in Mediterranean fruit flies: advantages for large and proteinfed males. Animal Behaviour, 58: 247-254.

Thornhill, R. \& J. Alcock, 1983. The evolution of insect mating systems. Harvard University Press, Cambridge, MA. 547p.

Whittier, T.S. \& K.Y. Kaneshiro, 1995. Intersexual selection in the Mediterranean fruit fly: does female choice enhance fitness? Evolution, 49: 990-996.

Whittier, T.S, F.Y. Nam, T.E. Shelly \& K.Y. Kaneshiro, 1994. Male courtship success and female discrimination in the Mediterranean fruit fly (Diptera: Tephritidae). Journal of Insect Behavior, 7: 159-170.

Whittier, T.S. K.Y. Kaneshiro \& L.D. Prescott, 1992. Mating behavior of editerranean fruit flies (Diptera: Tephritidae) in a natural environment. Annals of the Entomological Society of America, 85: 214-218.

Zucoloto, F.S., 1987. Feeding habits of Ceratitis capitata: can larvae recognize a nutritional effective diet? Journal of Insect Physiological, 33:349-353.

Zucoloto, F.S.; S. Puschel \& C.M. Message, 1979. Valor nutritivo de algumas dietas artificiais para Anastrepha obliqua (Diptera: Tephritidae). Boletim de zoologia São Paulo, 4: 75-80.

Zucoloto, F.S., 1988. Qualitative and quantitative competition for food in Ceratitis capitata. Revista Brasileira de Biologia, 48: $523-526$.

Recebido em: 29/o7/2011

Aceito em: 26/04/2012

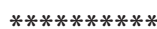

Como citar este artigo:

Silva Neto, A.M., V.S. Dias \& I.S. Joachim-Bravo, 2012. Comportamento Reprodutivo de Ceratitis capitata Wiedemann (Diptera: Tephritidae): Efeito do Tamanho dos Machos Sobre o seu Sucesso de Cópula. EntomoBrasilis, 5(3): 190-197.

Acessível em: http://www.periodico.ebras.bio.br/ojs/index.php/ebras/article/view/182
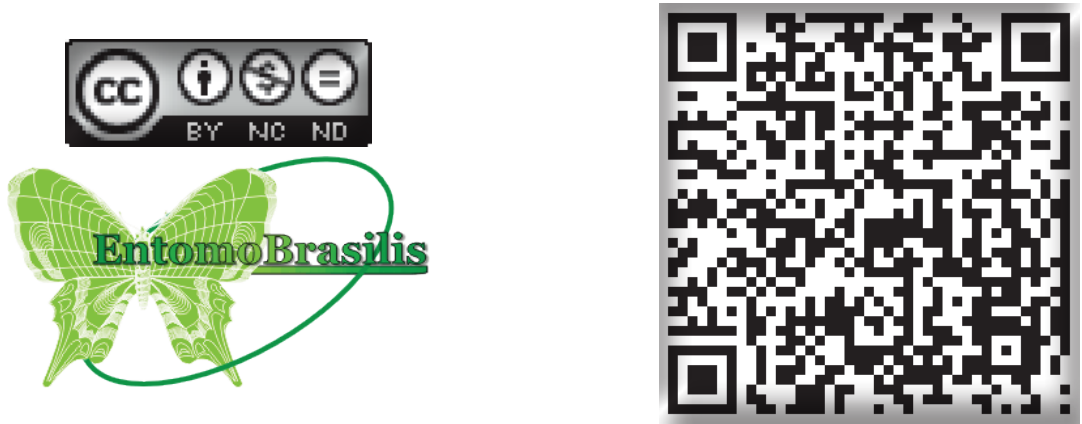